\title{
Inorganic Two-Dimensional Materials under Electron Irradiation: Stability, Evolution of the Atomic Structure, and Beam-Mediated Doping
}

\author{
Arkady V. Krasheninnikov \\ Department of Applied Physics, Aalto University, Helsinki, Finland
}

Isolation of a single sheet of graphene indicated that strictly two-dimensional (2D) materials can exist at finite temperatures. Indeed, inorganic 2D systems such as hexagonal BN sheets, transition metal dichalcogenides (TMD) with a common structural formula $\mathrm{MeX}_{2}$, where Me stands for transition metals (Mo, W, Ti, etc.), $\mathrm{X}$ for chalcogens ( $\mathrm{S}, \mathrm{Se}, \mathrm{Te}$ ), and $\mathrm{SiO}_{2}$ layers were later manufactured by various methods. All these materials have defects, which naturally affect their properties. Moreover, defects can appear during exposure to energetic electrons during sample examination in the transmission electron microscope (TEM) or deliberately be introduced by ion and electron irradiation to tailor the properties of the system [1].

In my talk, I will present the results of our first-principles theoretical studies of defects in inorganic 2D systems -- TMDs and silica obtained in collaboration with several TEM experimental groups [2-6]. I will also touch upon defect production in 2D systems under impacts of energetic electrons [2,3]. I will further discuss defect and impurity-mediated engineering of the electronic structure of 2D materials such as TMDs [3-4] and BN [7,8]. I will discuss mixed TMDs, such as $\mathrm{MoS}_{2 \mathrm{x}} \mathrm{Se}_{2(1-\mathrm{X})}$, which can be referred to as 2D random alloys [9]. Our simulations indicate that 2D mixed ternary random alloy $\mathrm{MoS}_{2} / \mathrm{MoSe}_{2} / \mathrm{MoTe}_{2}$ compounds are thermodynamically stable at room temperature, so that such materials can be manufactured by CVD or exfoliation techniques. Moreover, our simulations predicted that the direct gap in these materials can continuously be tuned depending on relative component concentration, as confirmed later on by several experimental groups.

I will finally touch upon defects in bilayer 2D silica [5,6] and show that the motifs of isolated point defects in this system and graphene [10] (two 2D structures with the same symmetry but otherwise very different properties) are similar, and include Stone-Wales-type defects formed by structural unit rotations, flower defects and reconstructed double vacancies. The morphology and energetics of extended defects, such as grain boundaries have much in common as well. As both $\mathrm{sp}^{2}$-hybridised carbon and bilayer silica can also form amorphous structures, our results indicate that the morphology of imperfect 2D honeycomb lattices is largely governed by the underlying symmetry of the lattice.

[1] A.V. Krasheninnikov and F. Banhart, Nature Materials, 6 (2007) p. 723.

[2] H.-P. Komsa, J. Kotakoski, S. Kurasch, O. Lehtinen, U. Kaiser, and A. V. Krasheninnikov, Phys. Rev. Lett. 109 (2012) 035503.

[3] H.-P. Komsa, S. Kurasch, O. Lehtinen, U. Kaiser, and A. V. Krasheninnikov, Phys. Rev. B 88 (2013) 035301.

[4] Y.-C. Lin, D.O. Dumcenco, H.-P. Komsa, Y. Niimi, A.V. Krasheninnikov, Y.-S. Huang, and K. Suenaga, Advanced Materials (2014) in press.

[5] T. Björkman, S. Kurasch, O. Lehtinen, J. Kotakoski, O.Yazyev, A. Srivastava, V. Skakalova, J. Smet, U. Kaiser, and A.V. Krasheninnikov, Scientific Reports 3 (2013) 3482.

[6] F. Ben Romdhane, T. Bjorkman, J.A. Rodriguez-Manzo, O. Cretu, A.V. Krasheninnikov, and F. Banhart, ACS Nano 7 (2013) 5175. 
[7] N. Berseneva, A. V. Krasheninnikov, and R.M. Nieminen, Phys. Rev. Lett. 107 (2011) 035501.4. [8] H.-P. Komsa, N. Berseneva, A. V. Krasheninnikov, and R.M. Nieminen, submitted.

[9] H.-P. Komsaand A. V. Krasheninnikov, J. Phys. Chem. Lett. 3 (2012) 3652.

[10] F. Banhart, J. Kotakoski and A. V. Krasheninnikov, ACS Nano, 5 (2011) 26. 